DOI: http://dx.doi.org/10.35381/racji.v4i7.391

\title{
La influencia de la concepción franco-hispana de la Jurisprudencia en Latinoamérica
}

\section{The influence of the Franco-Hispanic conception of Jurisprudence in Latin America}

\author{
Luis Oliverio Cañarte Mantuano \\ luiscanartemantuano@gmail.com \\ Universidad Laica Eloy Alfaro de Manabí \\ Ecuador \\ https://orcid.org/0000-0003-1666-7443 \\ Jacqueline Maria Lastenia Chiriboga Davalos \\ chiribogajackeline@gmail.com \\ Universidad Laica Eloy Alfaro de Manabí \\ Ecuador \\ https://orcid.org/0000-0003-2282-5823 \\ José Enrique Chávez Castillo \\ echavez@hotmail.es \\ Universidad Laica Eloy Alfaro de Manabí \\ Ecuador \\ https://orcid.org/0000-0003-0593-4614 \\ Gladys Maria Cedeño Delgado \\ abggladys@hotmail.com \\ Universidad Laica Eloy Alfaro de Manabí \\ Ecuador \\ https://orcid.org/0000-0002-9384-1380
}

Recibido: 23 de mayo de 2019

Aprobado: 14 de junio de 2019

\section{RESUMEN}

En esta comunicación se presenta una revisión bibliográfica de textos de autores como López (2011), Álvarez, (1864), Contreras, (1907) y Guzmán, (2006) acerca de la influencia de la concepción franco-hispana de la jurisprudencia en países latinoamericanos, se valora la jurisprudencia como base procesal del derecho, con un 
enfoque comparado en el marco latinoamericano. En América Latina la influencia proviene especialmente de Francia y España, donde se desconfiaba y se rechazaba la labor y el desempeño jurídico de los jueces. Posteriormente, con el paso paulatino, el desarrollo del tiempo y de la historia se incorporaron los términos: Doctrina legal y Jurisprudencia constante, lo cual incidió en el hecho de que la instauración y la aplicación de la jurisprudencia forense se abriera paso al reconocimiento del valor de la jurisprudencia en el derecho procesal, en las cortes de casación efectuadas en América Latina a imagen y semejanza del derecho franco-hispano.

Descriptores: Teoría legal; Derecho comparado; Régimen jurídico; Historia del derecho.

\section{ABSTRACT}

In this communication a bibliographic review of texts by authors such as López (2011), Álvarez, (1864), Contreras, (1907) and Guzmán, (2006) is presented about the influence of the Franco-Hispanic conception of jurisprudence in countries Latin Americans, jurisprudence is valued as the procedural basis of law, with a comparative approach in the Latin American framework. In Latin America the influence comes especially from France and Spain, where the work and legal performance of judges was distrusted and rejected. Subsequently, with the gradual step, the development of time and history the terms were incorporated: Legal Doctrine and Constant Jurisprudence, which influenced the fact that the establishment and application of forensic jurisprudence opened the way to the recognition of value of jurisprudence in procedural law, in cassation courts held in Latin America in the image and likeness of Franco-Hispanic law.

Descriptores: Legal theory; Comparative law; Legal systems; Legal history.

\section{INTRODUCCIÓN}

En el contexto internacional de América Latina, los Sistemas jurídicos han sido vistos como neorromanos, y positivistas por lógica filiación histórica de larga data, dado que en forma reiterada y clásica ya han adoptado y han creído que los jueces están solamente para aplicar la Ley, que los alegatos judiciales son meramente relevantes para describir normas solamente, cuando por la vía de la excepción éstas pueden ser asumidas como inciertas o poco claras. Por tanto, se acogen los supuestos de López (2011): quien refiere que: "La obligación judicial de fallar en correspondencia con el derecho solo se cumple a través de la obediencia a las reglas claras previamente establecidas por quien legisla". Aunado a ello, se asume que los jueces están irremediablemente atados a la Ley; sin embargo estos son independientes ante las 
sentencias judiciales con las cuales se ha fallado en casos ya procesados. En ese tenor, la Jurisprudencia ha sido históricamente confinada a ser considerada "como una fuente auxiliar del Derecho, que solo aplica en casos de silencio de la fuente primaria. Así las cosas, el derecho se enseña en las facultades universitarias como un conjunto complejo de reglas, principios y normas jurídicas principalmente de naturaleza legislativa que ha sido previamente codificada.

Por tanto, se advierte que el Derecho judicial es poco conocido, no se accede con facilidad a él; casi no hay ordenamiento ni sistematización de experiencias sobre el derecho y no se concede el justo valor que posee como base para el proceso del Derecho como tal. Ante la hegemonía del derecho codificado y legislado, la jurisprudencia de los tribunales de cierre de los sistemas latinos se ciñe, y se enmarca generalmente, a expandir fallos concretos. Desde este marco, la Jurisprudencia adquiere un valor determinado, que implica la potestad de cerrar definitivamente las contenciones jurídicas en torno a casos concretos que se presentan ante la judicatura. A la luz de estas consideraciones, se presenta este artículo que presenta la revisión analística de los trabajos de López (2011) Guzmán (2006), y Contreras (1907), entre otros sobre la influencia de la concepción europea, específicamente la francesa y la española sobre la jurisprudencia en la asunción del rol de los jueces en el derecho procesal latinoamericano.

Desde lo anteriormente expuesto, es preciso tomar en cuenta que, tradicionalmente en Ecuador, la Jurisprudencia fue vista como una fuente secundaria para consultar algunos casos específicos, para orientar las decisiones tomadas por los jueces, quienes se basan en las leyes; sin embargo, cuando entró en vigor la denominada Constitución de Montecristi se suscitó una evolución del derecho otorgando mayor valor y relevancia a la Jurisprudencia, posicionándose en la actualidad como base fundamental procesal de los casos, lo cual ha transformado el papel que desempeñan los jueces de la Corte Constitucional en la actualidad.

Sin embargo, al revisar la historia, se denota que en América Latina se adoptó un sistema denominado Continental Clásico Francés, en el marco del cual, amparados en el contexto jurídico, se tiene a la Ley como fuente principal de ordenamiento jurídico 
del Estado, configurándose la Constitución como la máxima Ley Estatal por excelencia por la que se rigen leyes, decretos, normativas y demás principios y estatutos.

Esta figura preconcebida acerca de la función del juez, empero, ha sido enfrentada y rechazada por diversos enfoques de teoría jurídica, los cuales han elaborado sus argumentos desde finales del siglo XIX hasta la actualidad, mismos que pueden asumirse, sin lugar a dudas, como los enfoques dominantes en la discusión contemporánea. En la actualidad puede observarse que en las cortes latinoamericanas el rol del juez ha quedado limitado a la solución de problemas, en menoscabo de ser intérpretes de la ley para promulgar las jurisprudencias pertinentes para actualizar el derecho en virtud de las decisiones a tomar en pertinencia con las exigencias sociales y jurídicas del entorno. De proseguirse con esta praxis inhibidora de la jurisprudencia, López (2011, p. 23), plantea lo siguiente:

i) Oscurecimiento de la naturaleza dialéctica de la argumentación judicial;

ii) Reducción de manera muy significativa de la transparencia de los argumentos $\mathrm{y}$ las complejidades en las que se hacen ponderaciones entre derechos e intereses enfrentados, negando tal complejidad y escondiéndola bajo el principio de legalidad;

iii) Socavación de la responsabilidad social y política que los jueces tienen que asumir en la toma de decisiones, en casos que son intrínsecamente difíciles o en los que el principio de legalidad no puede cerrar, por sí solo, las controversias sociales sobre la dirección y sentido en que el derecho debe resolver dichas situaciones;

iv) Formalización excesiva de la jurisprudencia de los órganos de cierre generando una hipertrofia del derecho de casación (o de otras expresiones de ritualismos procesales) en detrimento del derecho sustantivo y de su interpretación;

v) Invisibilización del papel de la Constitución y de los derechos fundamentales y humanos en la adjudicación contemporánea; y, finalmente, como consecuencia de todo lo anterior, 
vi) Se termina negando el papel efectivo que tiene la jurisprudencia como fuente del derecho y mecanismo post-legislativo de discusión y armonización social y política de criterios jurídicos intrínsecamente controversiales.

A la luz de las consideraciones precedentes, en este artículo se pretende, desde el reconocimiento del papel primordial que juega el principio de legalidad en el mantenimiento del Estado de Derecho, revisar analíticamente la incidencia europea y específicamente la francesa y la española en el solapamiento de la relevancia del rol de la Jurisprudencia en América Latina.

\section{El rol de los jueces, una mirada desde la concepción franco-jurídica heredada por la tradición jurídica Latinoamericana. Una visión histórica y comparada}

La asunción del liberalismo jurídico, en líneas generales, resultó evidente en el texto del Código Napoleón, así como en otros instrumentos legales generados en Europa, los cuales se basaban en el mismo fundamento ideológico, que posteriormente marcaron incidencia en la elaboración de los sistemas jurídicos y judiciales de las jóvenes repúblicas hispanoamericanas. En ese marco contextual, en lo referente al rol y visión de los jueces, el propio Código Civil francés del año 1804, exponía una perspectiva social y política contundente, la cual fue puesta de manifiesto en varias de sus disposiciones, en los artículos que se citan a renglón seguido:

a. Artículo 4: El juez que se niega a juzgar, bajo el pretexto del silencio, la oscuridad o la insuficiencia de la ley, puede ser procesado como culpable de denegación de justicia.

b. Artículo 5: Está prohibido que los jueces se pronuncien por disposición general y reglamentaria sobre los casos que se les presenten.

c. Artículo 6: No podemos derogar, por convenciones particulares, leyes que conciernen al orden público y la moral.

Desde la generación de tales artículos hasta su llegada a Latinoamérica se suscitó un proceso de influencia paulatino pero lapidario, dado que se configuraron en la fuente fundamental de la perspectiva de la labor de los jueces y su rol en el establecimiento de la Ley; esto fue permeando la legislación de estos países desde el transcurso de 
los siglos XIX y XX. Estableciéndose en primer orden, en las Islas del Caribe, tales como en Haití, en 1825, y luego la República Dominicana, en 1845, las cuales asumieron dogmáticamente el Código Francés como derecho nacional vigente. No obstante a ello, en ciertas regiones como en República Dominicana, la influencia fue más tardía, dado que ameritaron de 70 años (hasta 1884) cuando finalmente se obtuvo la traducción al idioma Castellano, de los artículos 4, 5 y 6, lo cual, se resume en lo que a continuación se expone:

a. Art. 4.- El juez que rehusare juzgar pretextando silencio, oscuridad 0 insuficiencia de la ley, podrá ser perseguido como culpable de denegación de justicia.

b. Art. 5.- Se prohíbe a los jueces fallar por vía de disposición general y reglamentaria las causas sujetas a su decisión.

c. Art. 6.- Las leyes que interesan al orden público y a las buenas costumbres no pueden ser derogadas por convenciones particulares.

A posteriori, se hicieron llegar a estos países, las versiones traducidas de la concepción que se tenía sobre la función judicial, en cuyo transcurso temporal se suscitaron sendos intentos de castellanización, adaptación o la denominada criollización del Legalismo Liberal Francés. De tal suerte lo describe Guzmán (2006: p. 45) Al referir que: "Después de estas traducciones más o menos directas del Code Civil Francés, en el Caribe, se inició a mediados del siglo XIX un proceso de codificación endógena en países como Perú (1852) y Chile (1855)".

De cualquier forma que se interpreten estas consideraciones de Guzmán (Op cit), en verdad, no hubo adecuación alguna del Código Civil Francés en el Caribe o en América Latina, dado que no se suscitaron diferenciaciones substanciales en cuanto a la perspectiva del rol del juez en el marco de la Ley; antes bien, estas traducciones y esfuerzos por adecuar el Código Civil a la región caribeño-latinoamericana, confirman en su interior, la asunción de que el Juez sólo está para aplicar la Ley y no para interpretarla.

Lo anteriormente expuesto ha sido reincidente en la ley latinoamericana, de tal suerte se demuestra en el Código Civil de Chile de 1855, el cual fu elaborado por don Andrés 
Bello y asumido a su vez, a posteriori, por varios países de la región, en este código se recopiló una perspectiva de la función judicial en la que, por una parte, se destacaba una enorme confianza en la capacidad de la ley de coordinar el comportamiento social y, por la otra, se evidenciaba una enfática antipatía frente al papel interpretativo de los jueces.

Para corroborarlo López (ibídem) refiere que: En el artículo 5 del Código Napoleón, se prohibía a los jueces "fallar por vía de disposición general y reglamentaria sobre las causas sujetas a su decisión". Incluso podría afirmarse, sin lugar a dudas, que el Código chileno demuestra con mayor claridad -si cabe- el sentido político de la prohibición suscitada en el estamento jurídico francés. En la concordancia de los tres primeros artículos que le integran, se configura un ejemplo típico y certero de la ideología liberal, legalista y antijudicialista de la cultura jurídica liberal de la postrevolución francesa, a saber:

a. Art. 1. La ley es una declaración de la voluntad soberana que, manifestada en la forma prescrita por la Constitución, manda, prohíbe o permite.

b. Art. 2. La costumbre no constituye derecho sino en los casos en que la ley se remite a ella.

c. Art. 3. Sólo toca al legislador explicar o interpretar la ley de un modo generalmente obligatorio.

Se evidencia a partir de lo precitado que la ley prevalece ante la costumbre y la jurisprudencia, a las que define como fuentes del derecho en potencial competencia y a las que pretende dominar.

\section{Disposiciones legales latinoamericanas a imagen y semejanza de su herencia franco-hispana}

En este sentido en razón de figurar la jurisprudencia en el ámbito latinoamericano, se reseña:

- El artículo 3 del Código Civil de El Salvador de 1859 se ordenó, tomando tan solo el primer inciso del artículo 3 del Código Bello, que "[s]ólo al legislador corresponde explicar o interpretar la ley de un modo generalmente obligatorio". La 
misma disposición se expresa en el artículo 3 del Código Civil de Honduras de 1880. Asimismo, en el artículo 17 del Código Civil de la Federación colombiana (de 1872) se establece que "[l]as sentencias judiciales no tienen fuerza obligatoria sino respecto de las causas en que fueron pronunciadas. Es, por tanto, prohibido a los jueces proveer en los negocios de su competencia por vía de disposición general o reglamentaria".

- Aunque en el contexto nicaragüense fue distinto el nivel de influencia también recibieron y aceptaron el clima ideológico general dominante en la época. La asunción de la ideología judicial francesa no requirió de la adopción de normas expresas: ella conformaba específicamente el ambiente cultural de la época que se convirtió en un determinante hegemónico, en el marco del cual, la jurisprudencia fue concebida con recelo como fuente del derecho y se esperaba que la función judicial funcionara de manera deductiva y mecánica con el propósito de salvaguardar la soberanía legislativa al tiempo que se extirpaba la posibilidad de arbitrariedad judicial.

De lo antes expuesto, se evidencia que fue el liberalismo jurídico francés- el que aunque no fue adoptado explícitamente (como sucedió en Nicaragua), se constituyó en la ideología judicial compartida por las élites jurídicas latinoamericanas del siglo XIX. De tal manera lo expresa López (ibídem) cuando afirma:

EI liberalismo jurídico y judicial del siglo XIX también lo ha sido de los siglos XX y del XXI. Constituye, por tanto, historia del presente que todavía determina, en buena medida, el papel y la función de los jueces latinoamericanos y, en especial, de sus cortes de cierre. En los países donde se adoptó la prohibición de que los jueces fallaran por vía general y reglamentaria (p. ej., en Haití, República Dominicana, Chile, El Salvador, Honduras, Ecuador y Colombia) hay que reparar que tales textos son todavía derecho vigente que han soportado admirablemente los embates del tiempo y los cambios en ideología sobre la función judicial. En los países donde la norma no se adoptó expresamente, la teoría liberal de la adjudicación es ideología todavía actuante y dinámica en la manera de comprender efectivamente el derecho. Pero esta es, en realidad, tan solo la primera mitad de la historia. (p. 37) 


\section{Concepción de la Jurisprudencia en Francia y su influencia en América Latina}

La revisión pretérita evidencia una perspectiva jurídica influyente, distinguida como el enfoque anti-jurisprudencial y anti-judicialista del legocentrismo francés que fue cambiando con el transcurrir del tiempo dentro del propio continente europeo. A mediados del siglo XIX los franceses pusieron de relieve su reconocimiento al valor cognitivo de la denominada jurisprudence constante que los tribunales iban formando con sus fallos; este mismo hecho en España recibió la denominación de "doctrina legal".

Esta escuela legal causó la ascensión específica de la apertura de legalidad que determinaba los casos a disponer podían ser determinados por el juez exclusivamente en precisa y fiel acatamiento de alguna disposición legal. No obstante a ello, se puso en evidencia que, muchos casos no podían resolverse con referencia a la ley: se puso de manifiesto que los códigos tenían muchos más vacíos, ambigüedades, contradicciones y choques de intereses y derechos de lo que establecía el ideal ilustrado de la codificación comprendida por todos.

Pese a la aversión a la revolución francesa, ante la valoración de la jurisprudencia se abría paso la evolución del derecho hacia contextos inesperados por el legislador, hacia los variados elementos contenciosos y polémicos que la experiencia forense iba aperturando con el tiempo.

Poco a poco la jurisprudencia fue ganando terreno rechazando la resolución de los casos únicamente con el enfoque legalista y la mera exégesis de textos, en este sentido, López (2011), plantea que "empezaba a aceptarse con lentitud que la jurisprudencia podía, en primer lugar, suplir criterios para casos que no estaban regulados explícitamente y, en segundo lugar, crear derecho sin que ello significara usurpar indebidamente la función legislativa" (p. 39).

Esta constatación permitiría muy lentamente una cierta rehabilitación del valor normativo y del significado político de la jurisprudencia. Poco a poco se fue regenerando una fuerza normativa de la jurisprudencia fue entonces cuando en Europa se publican "colecciones" o "compendios" de jurisprudencia que, paulatinamente, se convirtieron en fuente indispensable en el trabajo diario de los 
abogados y de los jueces. La praxis forense de la jurisprudencia evoluciona significativamente de la mano de la amplia disponibilidad que las sentencias empiezan a tener con estas formas de difusión. En el marco contextual francés se publicó;

Desde el año de 1827 el Recueil de sentencias de Dalloz, con el título de "Jurisprudence générale du Royaume: en matière civile, commerciale et criminelle ou journal des audiences de la cour de cassation et descours royales". Posteriormente este mismo autor publicó en 1835 el Dictionnaire général et raisonné de législation, de doctrine et de jurisprudence (López, 2011).

En este texto, al autor expone, desde el exordio de esta obra, que era muy complejo para los juristas de la época atinar con las soluciones adecuadas a los casos que se les presentaban debido a la creciente dispersión de opiniones jurisprudenciales y doctrinales. Tal situación puso de relieve que desde el inicio del siglo XIX, se expresaba una clara ideología legalista de la codificación francesa. Álvarez (1864), reprende a la cultura jurídica nacional y expresa:

En nuestros archivos se encuentran doctrinas preciosas, decisiones admirables, en que resalta un criterio jurídico tan exacto y tan perfecto, que para nada necesita ir a mendigarse del foro francés -el más justamente afamado sin duda; pero todo yace en el olvido más deplorable, si ya no es también en la incuria i en el desgreño más completos. Obtenida una decisión en un negocio cualquiera, surte sus efectos de actualidad... i luego va a confundirse en el voluminoso caudal de expedientes de un archivo, sin dejar la más pequeña huella luminosa para que encamine al litigante que viene después, o aparte del mal sendero al que vaya extraviado, o contenga las pretensiones absurdas o temerarias del ignorante y malicioso, u obligue al juez voluntarioso a mantener i respetar las tradiciones de la lei. (p.40)

La propuesta de Álvarez evidencia que algunos jueces latinoamericanos de vanguardia estaban conectados con los cambios que sufría el valor de la jurisprudencia en la Europa de la época. El valor del rol de la jurisprudencia llegó a América Latina a través de la nueva función que debían asumir las cortes supremas de la región cuando se convirtieron en cortes de casación, emulando el modelo de la ley procesal civil española. "Las cortes supremas de América Latina fueron asumiendo lentamente su función de Cortes de Casación en el último cuarto del siglo XIX y en los primeros años del siglo XX". La "doctrina legal" española, en contraposición a la 
jurisprudence constante de los franceses, logró adquirir reconocimiento normativo explícito y no solamente el cultural y profesional que ahora se reconocía a los célebres arrêts franceses. En muchos países, los arrêts de la Cour de Cassation se emplearon directamente como doctrina legal en la solución de los casos contenciosos.

La adopción de un modelo jurisprudencial en dependencia del modelo jurista de Francia es marcada y notoria, en el caso de la Suprema Corte de República Dominicana. En ese marco, se podría considerar que ello se configuró en una característica común del derecho de América Latina y, en especial, de América Central, se asumió en forma prácticamente generalizada de que la jurisprudencia tiene valor como fuente de derecho bajo la forma de la "doctrina legal". La concepción de "doctrina legal" fue adoptada por los legisladores latinoamericanos, ya no del derecho francés, sino específicamente del ordenamiento procesal español.

En ese marco, los textos legales de 1855 expresan en forma clara, como lo enfatiza Contreras, que el sintagma "doctrina legal" no aludía exactamente a las decisiones de los jueces sino a los principios u opiniones (cualquiera que fuera su fuente) que ellos "admitieran" o "recibieran". He aquí un ejemplo de este modo de empleo de ello, en una sentencia española de 1867 que a renglón seguido se presenta:

Es doctrina legal y admitida por la jurisprudencia la que procede de la excepción de cosa juzgada, aun cuando en el segundo pleito que se proponga se haya variado el nombre de la acción deducida en el primero, siendo idénticos el fundamento de ambos su objeto y la causa ó razón de pedir; en el supuesto de que, concurriendo los demás requisitos legales, se haya decidido también el pleito en que recayó la ejecutoria por el mismo fundamento y no por otro diferente. (p. 801)

En atención a lo antes expuesto, los términos "doctrina legal" y "doctrina jurisprudencial" no poseían al principio un valor sinonímico en cuanto a semántica se refiere, aunque con el tiempo y el uso adquirieron connotaciones similares. En ese orden se resalta que el reconocimiento en España de la "doctrina legal" se puede interpretar, a decir de Contreras (1907): "desde la perspectiva de la superación del legalismo a ultranza propio de los primeros intentos codificadores". En ese escenario, el recurso de nulidad responde a la concepción de que la ley es perfecta y completa, y que mediante su aplicación se pueden resolver todos los casos posibles "sin que el 
juez pueda interpretarla sino sólo aplicarla automáticamente: no hay en él, consecuentemente, lugar para regla subsidiaria alguna".

\section{La concepción de la denominada "doctrina legal” adoptado en Latinoamérica: se instaura el poder jurisprudencial de las Cortes Supremas en la Región}

El sustento legal español del siglo XIX fue la base asumida en el derecho latinoamericano adoptado desde una concepción de doctrina legal. La adopción se asumió tanto en cuanto a las normas de procedimiento civil, como en las legislaciones típicamente autóctonas de amparo de derechos fundamentales que se expandían también desde el siglo XIX por todo México y en la América Central. La doctrina legal y su concepción específica de relación con la jurisprudencia fueron recibidas en América Latina desde la década de 1880 casi en toda esta vasta región. De esta manera lo explica López (ibídem):

La jurisprudencia podía hacer las veces de la "ciencia" que reclamaba el positivismo; el orden resultante era la vinculación de todos los jueces a la "doctrina" jurisprudencial y científica, a la vez, que emanaba de los tribunales. Esta justificación política es particularmente cierta en los casos de México y Colombia donde sus Supremos adoptaron la jurisprudencia vinculante como mecanismo explícito para asegurar la unidad política del país frente a los conatos de un federalismo judicial salido de cauce. (p.40)

En esta cita el autor refiere que las Cortes Supremas de Justicia de Colombia y México, por ejemplo, asumieron la jurisprudencia vinculante como un procedimiento lógico para preservar la unidad del poder judicial en tales países ante lo que consideraban los embates políticos de los intentos fallidos del federalismo por instaurarse en aquella época.

Estas nuevas leyes importadas, en correspondencia con este último enfoque, no perseguían el propósito de resolver nada, sino que se ceñían a imitar los modelos que les resultaban prestigiosos por motivos alejados de la institucionalidad. Los ejemplos expuestos suscitados en México y Colombia, al parecer, tenían una causa política basada en la necesidad de controlar el federalismo; empero, resulta obvio que "la pura influencia de la por entonces nueva casación española se hizo notar de forma determinante para permitir así la construcción de muy pesados poderes de ordenación 
jurisprudencial en sus respectivas cortes supremas". Desde una u otra perspectiva teórica, se hacía evidente "que las supremas cortes regionales estaban asumiendo poderes de ordenación jurisprudencial en contra de la visión clásica de la exégesis y del legocentrismo francés".

\section{Referentes metodológicos esenciales}

En este artículo, se parte de un hecho real, vale decir, la Evolución en un periodo histórico determinado- de la jurisprudencia en el derecho latinoamericano desde la influencia recibida en esta región por las concepciones europeas. En ese tenor, se asume lo propuesto por (Bunge, 200: p. 19), quien refiere que: "el método científico se alimenta de valiosas características como son: los enunciados fácticos (...), conjeturas, suposiciones, presunciones".

La presente investigación es de carácter Documental Bibliográfica; por cuanto fueron los libros y artículos existentes sobre el tema la principal fuente de consulta. Hizo revisión de cuerpos normativos como: la Constitución de la República, artículos y libros relacionados con el tema de investigación. También se abordó doctrina y jurisprudencia desde un enfoque franco-hispano para analizar su influencia y sustrato en las concepciones y perspectiva del derecho y la jurisprudencia latinoamericana en general.

Con la utilización del enfoque metodológico de investigación histórico y comparado se pretendió analizar y determinar si existe una diferenciación evolutiva entre el sistema del derecho y la jurisprudencia desarrollada en el tiempo con la corriente del pensamiento filosófico más conocido en diversos países de América Latina, como el positivismo. Se intentó valorar el proceso evolutivo de desarrollo de la jurisprudencia a través del tiempo y desde su influencia europea en esta región del mundo. Este método permite apreciar que los diversos fenómenos no se presentan de manera casual, sino que es el resultado de un largo proceso histórico que los origina, motiva o da lugar a su existencia.

El análisis de un objeto significa: "comprender la revisión de sus características a través de las partes que la integran, es hacer una separación de sus componentes y 
observar periódicamente cada uno de ellos," a los fines de identificar tanto su dinamia particular como las relaciones de correspondencia que guardan entre sí y dan origen a las características generales que se quieren conocer. Al estudiar cada artículo científico y legal se hizo uso de este método.

Por la naturaleza de la presente investigación las técnicas utilizadas fueron las siguientes: Para la recolección de la información, se utilizó ésta técnica que consistió en ir tomando nota de toda la información obtenida en fichas, tal como sigue a continuación:

a. Fichas Nemotécnicas. Tarjetas, que poseen medidas estándar, en ella se registra la información obtenida de la lectura.

b. Fichas Bibliográficas. Tarjetas, que poseen medidas estándar, sirven para identificar, las fuentes de información bibliográfica, que consisten en libros, autores, etc.

c. Fichas Hemerográficas. Tarjetas, que poseen medidas estándar, en ella se registra la información obtenida de la investigación de revistas, periódicos y demás publicaciones.

\section{DISCUSIÓN Y ANÁLISIS DE LOS RESULTADOS}

Una vez revisado los apuntes y notas registradas en las fichas bibliográficas puede analizarse que la jurisprudencia como fuente de derecho, ha tenido gran relevancia y ha evolucionado en el tiempo. En América Latina la influencia proviene directamente de Europa, especialmente de países como Francia y España, donde se desconfiaba y se rechazaba la labor y el desempeño jurídico de los jueces. Posteriormente con el paso paulatino, el desarrollo del tiempo y de la historia se incorporaron los términos: Doctrina legal y Jurisprudencia Constante por diversas razones, lo cual incidió en el hecho de que mediante la instauración y la aplicación de la jurisprudencia forense se abriera paso al reconocimiento del valor de la jurisprudencia en el derecho procesal, en las cortes de casación efectuadas en América Latina a imagen y semejanza de derecho franco-hispano. 
Estos conceptos, configuraron apenas el campo en donde se desarrollaron sendas paradojas y contradicciones en la región. Por una parte, se suscitó la necesidad de lograr convergencia entre los criterios interpretativos en una misma jurisdicción con el reclamo y Por otra parte, se hizo evidente la defensa enfática, que se ha hecho de la autonomía adjudicativa de los jueces y de su exclusiva dependencia y responsabilidad frente a la ley. En ese orden de las coincidencias hay que afirmar que la jurisprudencia pueda establecer reglas primarias de conducta ex ante; a pesar de ello, ya iniciado el pleito judicial, ex post si se quiere, se usa el argumento basado en la jurisprudencia con innegable frecuencia.

Actualmente puede afirmarse que, existe en muchos países de la región latinoamericana que se ha suscitado una prominente relevancia de la jurisprudencia vinculada al proceso judicial y sus múltiples elementos factoriales, relativas a la casación, a sus causales y a las formas cómo los abogados deben estructurar el recurso.

En ese orden, López (2011) resalta que la "jurisprudencia de los jueces parece ensimismada y autorreferencial: se mira su propio ombligo tratando de definir, a veces con exasperante detalle, la interpretación de normas procesales y casacionales".

En consideración a los aspectos antes expuestos, cabría exponer la siguiente interrogante: ¿Posee o no la jurisprudencia valoración significativa fuente de derecho? Con respecto a este tópico, en los países de Latinoamérica, se ha evidenciado de un tiempo a esta parte que realmente se ha resaltado la jurisprudencia: basta referir este tema en el mundo jurídico con jueces y abogados de diferentes jurisdicciones para asumir y comprender entre líneas que, los distintos expresiones de los enfoques del derecho en diversas naciones se han constituido en sendas manifestaciones nacionales de un tronco jurídico-ideológico común.

\section{CONCLUSIONES}

Una primera conclusión a la que puede llegarse radica en lo siguiente: América Latina dio el paso hacia el cambio, emulando el modelo jurídico europeo, y modificó su concepción demostrando al principio un rechazo y una desconfianza contundentes en 
torno al manejo de la justicia y el derecho por los jueces, hasta llegar a la asunción posterior de un "judicialismo" y un "jurisprudencialismo" que ya parece estar bien formado en el viejo continente a mediados del siglo XIX y que se comienza a recibir en América a finales del mismo.

Este tránsito hacia el "jurisprudencialismo" se nota con toda claridad, como marca de época, en los libros, comentarios y sentencias que cruzaban el Atlántico y, posteriormente, en la doctrina científica del derecho que empiezan a escribir las élites jurídicas criollas hacia 1880. Lo que sirvió como fundamento para la consolidación de las Cortes Supremas de Justicia latinoamericanas en esa misma fecha, como resultado evidente de la denominada doctrina legal en el marco del enjuiciamiento civil de España.

En esta recepción del modelo español se evidencia la influencia del derecho europeo en las culturas jurídicas locales que todavía no se habían liberado completamente del trauma de la dependencia colonial. Esta influencia de las ideas españolas, coincidió quizá azarosamente con la emergencia de la centralización del poder en países con enfoque federalista o regionalista que amenazaban la estabilidad nacional. Aunado a ello, en la cultura general, "las corrientes del positivismo científico de finales del siglo XIX estuvieron también relacionadas con el renacimiento del valor de la jurisprudencia, a la que le daba la estatura de doctrina científica en el campo del derecho".

Este conglomerado de elementos multifactoriales forman parte de una explicación parcial de las causas por las cuales el jurisprudencialismo europeo impactó de manera significativa en el derecho local de Hispanoamérica porque explican de manera suficiente cuáles fueron las líneas estructurales de una convergencia que se nota todavía al día de hoy en los conceptos, usos, prácticas y teorías del derecho en la región.

Como conclusión final, podría decirse que en los sistemas jurídicos actualmente vigentes en varios países de Latinoamérica pueda que se susciten algunas diferenciaciones mínimas pero se observa una génesis y un modelo común de desarrollo en el tema de la Jurisprudencia como fundamento del Derecho procesal penal en América Latina y El Caribe, lo cual implica desde la visión de Chirino \& 
Giménez (2019), trabajar en determinar la jurisprudencia desde un estudio sistemático y continuo de los recursos jurídicos por parte del juez, esto involucra diseñar un circulo analítico que permita transcender el modelo que solo limita a generar decisiones, lo cual proyecta la posibilidad de estar en consonancia con un modelo latinoamericano acorde a las exigencias del contexto socio jurídico.

\section{REFERENCIAS CONSULTADAS}

1. Álvares, S. (1864) "Decisiones judiciales o estudios sobre el derecho patrio". Bogotá, Biblioteca Nacional de Colombia, Fondo Pineda n. . 815, Folio 40, 1864. En Teoría impura del derecho: la transformación de la conciencia jurídica latinoamericana (Bogotá, Legis, segunda reimpresión, 2005, capítulo 4).

2. Bunge, M., (2003), La Ciencia su método y su filosofía, ISBN: 958-33-3344-1 Colombia: Edit. Fundación Promotora Colombiana de Cultura, pág. 117.

3. Carbonell, M. (2007), El Neoconstitucionalismo en su Laberinto, España, Edit. Madrid, pág. 86.

4. Contreras, P. (1907) "Problemas actuales de la casación foral en Aragón". Revista de Derecho Civil Aragonés III, n. ${ }^{\circ}$ 2. "Ley de enjuiciamiento civil de 3 de febrero de 1881 concordada y anotada con gran extensión según la doctrina de los autores a jurisprudencia del Tribunal Supremo por la redacción de la Revista General de Legislación y Jurisprudencia bajo la dirección de D. Emilio Reus". Madrid, Hijos de Reus editores, 2. ㄹ edición, tomo I, 1907, p. 801. Umbral

5. Chirino, L., \& Giménez, M. (2019). Conducta criminal y su relación con la imputabilidad como elemento del delito. IUSTITIA SOCIALIS, 4(6), 28-51. doi:http://dx.doi.org/10.35381/racji.v4i6.287

6. Cruz, R., (2006), La Historia de la política del movimiento social, ISBN: 1575, Derechos de autor 0361. España: Edit. Complutense "RDL", págs. 94-95.

7. Fon, V., Parisi, F (2004) "Judicial Precedents in Civil Law Countries: A Dynamic Analysis”. George Mason University. Law and Economics Research Paper n.os 04-15 (abril 2004).

8. Glenn, H. P. Legal Traditions of the World. Londres, Oxford, 2000, pp. 226-229.

9. Guzmán, F. (2006) Historia de la codificación civil en Iberoamerica. Aranzadi. Madrid, España. 
10. Jellinek, G. (1978), Teoría General del Estado, Argentina, Edit. Albatros, Buenos Aires, pág. 90.

11. Laski, H. J. (1984), El liberalismo, México, Fondo de cultura económica, pág. 88

12. López, D. (2011) La jurisprudencia como fuente del derecho. Visión histórica y comparada. Revista de derecho Constitucional Umbral № 1, enero-junio 2011, pp. 21-54. ISSN 1390-6615. Corte Constitucional del Ecuador. Centro de Estudios y Difusión del Derecho Constitucional, Quito, Ecuador

13. López, R. (2002). La jurisprudencia y su función complementaria del ordenamiento jurídico (Madrid, Civitas, 2002, pp. 69 a 100)

14. Montesquieu; (1980), El Espíritu de las Leyes, España, Edit. Tecnos, pág. 103.

15. Peces, J. (2001). "Valor de la jurisprudencia" publicado en el libro La fuerza vinculante de la jurisprudencia, editado por Joaquín Samper Juen (Madrid, Consejo General del Poder Judicial, 2001, pp. 31 a 48).

16. Peter, C. (1999) Comparative Law in a Changing World. Cavendish: Londres, Inglaterra.

17. Sánchez, L. (1947), Lecciones de Derecho Político, España, Edit. Granada Traveset, pág. 102.

18. Vega, F. (1883) "La nueva ley de amparo de garantías individuales. Comentarios acerca de sus disposiciones más importantes. Ensayo crítico filosófico de la ley”. México, Imprenta de J. Guzmán, 1883, p. 249.

19. Verdú, P. (2001), Estado liberal de derecho y estado social de derecho, España, Edit. Universidad Complutense de Madrid, pág. 87.

\section{REFERENCES CONSULTED}

1. Álvares, S. (1864) "Judicial decisions or studies on national law". Bogotá, National Library of Colombia, Pineda Fund No. 815, Folio 40, 1864. In Impure Theory of Law: The Transformation of Latin American Legal Consciousness (Bogotá, Legis, Second Reprint, 2005, Chapter 4).

2. Bunge, M., (2003), Science its method and its philosophy, ISBN: 958-33-33441 Colombia: Edit. Colombian Promotora Foundation of Culture, p. 117. 
3. Carbonell, M. (2007), The Neoconstitutionalism in its Labyrinth, Spain, Edit. Madrid, p. 86.

4. Contreras, P. (1907) "Current problems of the cassation in Aragon". Aragonese Civil Law Magazine III, No. 2. "Civil Procedure Law of February 3, 1881, agreed and annotated to a great extent according to the doctrine of the authors to jurisprudence of the Supreme Court by the wording of the General Journal of Legislation and Jurisprudence under the direction of D. Emilio Reus ". Madrid, Children of Reus editores, 2nd edition, volume I, 1907, p. 801. Threshold

5. Chirino, L., \& Giménez, M. (2019). Criminal conduct and its relationship with imputability as an element of crime. IUSTITIA SOCIALIS, 4 (6), 28-51. doi: http: //dx.doi.org/10.35381/racji.v4i6.287

6. Cruz, R., (2006), The History of the politics of the social movement, ISBN: 1575 , Copyright 0361. Spain: Edit. Complutense "RDL", p. 94-95.

7. Fon, V., Parisi, F (2004) "Judicial Precedents in Civil Law Countries: A Dynamic Analysis”. George Mason University. Law and Economics Research Paper No. 04-15 (April 2004).

8. Glenn, H. P. Legal Traditions of the World. London, Oxford, 2000, pp. 226-229.

9. Guzmán, F. (2006) History of civil coding in Latin America. Aranzadi Madrid Spain.

10. Jellinek, G. (1978), General Theory of the State, Argentina, Edit. Albatros, Buenos Aires, p. 90.

11. Laski, H. J. (1984), Liberalism, Mexico, Economic Culture Fund, p. 88

12.López, D. (2011) The jurisprudence as a source of law. Historical and comparative vision. Journal of Constitutional Law Threshold No. 1, JanuaryJune 2011, pp. 21-54. ISSN 1390-6615. Constitutional Court of Ecuador. Center for Studies and Dissemination of Constitutional Law, Quito, Ecuador

13. López, R. (2002). The jurisprudence and its complementary function of the legal system (Madrid, Civitas, 2002, pp. 69 to 100)

14. Montesquieu; (1980), The Spirit of the Laws, Spain, Edit. Tecnos, p. 103.

15. Fish, J. (2001). "Value of jurisprudence" published in the book The binding force of jurisprudence, edited by Joaquín Samper Juen (Madrid, General Council of the Judiciary, 2001, pp. 31 to 48). 
16. Peter, C. (1999) Comparative Law in a Changing World. Cavendish: London, England.

17. Sánchez, L. (1947), Lessons in Political Law, Spain, Edit. Traveset grenade, p. 102

18.Vega, F. (1883) "The new law for the protection of individual guarantees. Comments about its most important provisions. Critical philosophical essay of the law ". Mexico, J. Guzmán Press, 1883, p. 249

19. Verdú, P. (2001), Liberal State of Law and Social Status of Law, Spain, Edit. Complutense University of Madrid, p. 87.

(C2019 por los autores. Este artículo es de acceso abierto y distribuido según los términos y condiciones de la licencia Creative Commons Atribución-NoComercial-Compartirlgual 4.0 Internacional (CC BY-NC-SA 4.0)

(https://creativecommons.org/licenses/by-nc-sa/4.0/). 